\title{
Lifting the curtain: The Seyfert galaxy Mrk 335 emerges from deep low-state in a sequence of rapid flare events
}

\author{
S. Komossa ${ }^{1}$, D. Grupe ${ }^{2}$, L. C. Gallo ${ }^{3}$, P. Poulos ${ }^{2}$, D. Blue ${ }^{3}$, E. Kara ${ }^{4}$, G. Kriss ${ }^{5}$, A. L. Longinotti ${ }^{6}$, \\ M. L. Parker ${ }^{7}$, and D. Wilkins ${ }^{8}$ \\ 1 Max-Planck-Institut für Radioastronomie, Auf dem Hügel 69, 53121 Bonn, Germany \\ e-mail: astrokomossa@gmx.de \\ 2 Dept. of Physics, Earth Science, and Space System Engineering, Morehead State University, 235 Martindale Dr, Morehead, KY \\ 40351, USA \\ 3 Department of Astronomy and Physics, Saint Mary's University, 923 Robie Street, Halifax, NS B3H 3C3, Canada \\ ${ }^{4}$ MIT Kavli Institute for Astrophysics and Space Research, Cambridge, MA 02139, USA \\ 5 Space Telescope Science Institute, 3700 S. Martin Drive, Baltimore, MD 21218, USA \\ ${ }^{6}$ Instituto Nacional de Astrofísica, Óptica y Electrónica INAOE- CONACyT, Luis E. Erro 1, Tonantzintla, 72840 Puebla, Mexico \\ 7 European Space Agency (ESA), European Space Astronomy Centre (ESAC), 28691 Villanueva de la Canada, Madrid, Spain \\ 8 Kavli Institute for Particle Astrophysics and Cosmology, Stanford University, 452 Lomita Mall, Stanford, CA 94305, USA
}

Received 3 August 2020 / Accepted 28 September 2020

\begin{abstract}
Context. The narrow-line Seyfert 1 galaxy Mrk 335 was one of the X-ray brightest active galactic nuclei, but it has systematically faded since 2007.

Aims. We report the discovery with Swift of a sequence of bright and rapid X-ray flare events that reveal the emergence of Mrk 335 from its ultra-deep multiyear low state.

Methods. Results are based on our dedicated multiyear monitoring of Mrk 335 with Swift.

Results. Unlike other bright active galactic nuclei, the optical-UV is generally not correlated with the X-rays in Mrk 335 on a timescale of days to months. This fact either implies the absence of a direct link between the two emission components; or else implies that the observed X-rays are significantly affected by (dust-free) absorption along our line of sight. The UV and optical, however, are closely correlated at the $99.99 \%$ confidence level. The UV is leading the optical by $\Delta t=1.5 \pm 1.5 \mathrm{~d}$. The Swift X-ray spectrum shows strong deviations from a single power law in all brightness states of the outbursts, indicating that significant absorption or reprocessing is taking place. Mrk 335 displays a softer-when-brighter variability pattern at intermediate X-ray count rates, which has been seen in our Swift data since 2007 (based on a total of 590 observations). This pattern breaks down at the highest and lowest count rates.

Conclusions. We interpret the 2020 brightening of Mrk 335 as a decrease in column density and covering factor of a partial-covering absorber along our line of sight in the form of a clumpy accretion-disk wind that reveals an increasing portion of the intrinsic emission of Mrk 335 from the disk and/or corona region, while the optical emission-line regions receive a less variable spectral energy distribution. This then also explains why Mrk 335 was never seen to change its optical Seyfert type (not "changing look") despite its factor $\sim 50 \mathrm{X}$-ray variability with Swift.
\end{abstract}

Key words. galaxies: active - galaxies: nuclei - galaxies: individual: Mrk 335 - X-rays: galaxies - galaxies: Seyfert quasars: supermassive black holes

\section{Introduction}

Active galactic nuclei (AGN) in extreme minima or maxima states provide us with important insight into the physics of the black hole and accretion disk region. The group of narrow-line Seyfert 1 galaxies (NLS1 hereafter) is known to exhibit particularly strong flux and spectral variability in X-rays (reviews by Gallo 2018 and Komossa 2018, and references therein). Mrk 335 (Markarian 1977) is a member of the class of NLS1 galaxies at redshift $z=0.0258$ with a black hole mass of $M_{\mathrm{BH}}=2.7 \times$ $10^{7} M_{\odot}$ (Grier et al. 2012). Its optical spectrum is characterized by strong high-ionization coronal emission lines with ionization states of up to [FeX] (Grupe et al. 2008) that imply the presence of a strong EUV-X-ray continuum. Mrk 335 was detected as a bright and variable UV emitter in early International Ultraviolet Explorer (IUE) and Hubble Space Telescope (HST) observations (e.g., Dunn et al. 2006; Longinotti et al. 2013).

For a time, this was one of the X-ray brightest AGN (Tananbaum et al. 1978; Pounds et al. 1987; Grupe et al. 2001; Bianchi et al. 2001). Then the X-ray flux of Mrk 335 dropped dramatically in 2007 (Grupe et al. 2007, 2008). Multiple epochs of exceptional flaring and dipping on its way to and beyond this low state motivated several deeper spectroscopic observations with XMM-Newton, Suzaku, NuSTAR, and the HST (Grupe et al. 2012; Gallo et al. 2013, 2015, 2018, 2019; Kara et al. 2013; Longinotti et al. 2013, 2019; Parker et al. 2014; Wilkins et al. 2015; Wilkins \& Gallo 2015; Komossa et al. 2017; Choudhury et al. 2019; Parker et al. 2019). These observations 
have shown that Mrk 335 is well fit with varying contributions of blurred reflection and (ionized) absorption. The reflection model explains diminished X-ray continuum emission with changes in a corona that has collapsed in toward the black hole, and sometimes forms a collimated outflow in X-ray flare states. The absorption model explains several epochs of flares and fades with changes in ionization state and covering factor of the absorbers, which were detected with the $X M M-N e w t o n$ reflection grating spectrometer (RGS) and in the UV with the HST.

We report the emergence of Mrk 335 from its recent ultradeep low state (which lasted from late 2017 to at least January 2020), based on 45 new observations with the Neil Gehrels Swift observatory (Gehrels et al. 2004) each in all seven wavebands from optical to X-rays. These are part of our long-term monitoring of Mrk 335, which started in May 2007. When Mrk 335 was observed on 2020 May 16, it was found already at a flux level that exceeded all observations since February 2018. In particular, it was very bright in the UV as well and appeared at a similar UV flux level as seen last during the flare at the end of 2011. Because of this outburst in May-June 2020, we started an intensive monitoring campaign with Swift, and we report the results here. We use a cosmology (Wright 2006) with $H_{0}=70 \mathrm{~km} \mathrm{~s}^{-1} \mathrm{Mpc}^{-1}$, $\Omega_{\mathrm{M}}=0.3$ and $\Omega_{\Lambda}=0.7$ throughout this paper.

\section{Data analysis}

\subsection{Swift XRT}

We have monitored Mrk 335 with Swift for more than 13 years since May 2007 with a cadence of typically 1-14 days, depending on the brightness and variability state of Mrk 335. Long gaps of several months occur each year when Mrk 335 is unobservable with Swift because of its proximity to the Sun. Mrk 335 came out of the Swift Sun constraint on 16 May 2020 (Grupe et al. 2020) and we resumed our monitoring (Figs. 1 and 2). The Swift X-ray telescope (Burrows et al. 2005) was operating in photoncounting mode (Hill et al. 2004) with typical exposure times of 1-2 ks (Table A.1).

X-ray count rates were determined using the online XRT product tool at the Swift data centre in Leicester (Evans et al. $2007)^{1}$. For a dedicated spectral analysis of select epochs, source photons were extracted within a circle of radius 59", and the background was determined in a nearby circle with a radius of $236^{\prime \prime}$. During the faintest states, spectral fitting of individual spectra is not possible, and we instead measured hardness ratios, defined as $H R=$ hard-soft hard+soft where soft and hard are the counts in the $0.3-1.0$ and $1.0-10 \mathrm{keV}$ energy bands, respectively. $H R$ was determined by applying the Bayesian estimation of hardness ratios, BEHR (Park et al. 2006) ${ }^{2}$.

In order to obtain a high-state spectrum for spectral fitting of a better signal-to-noise ratio (S/N), we combined the 2020 June 23 - July 1 Swift data when Mrk 335 was brightest (Target ID 13544, segments 011-016). For comparison, we created a lowstate spectrum when Mrk 335 was in its lowest state with data from May 2019 (Target ID 33420, segments 218-221). These are the two primary data sets used for spectral fitting. In both cases we created new ancillary response files (arfs) by adding the arfs of the single spectra weighted by their exposure time using the FTOOL command addarf. The coadded X-ray spectra in the band (0.3-10) $\mathrm{keV}$ were then analyzed with the software package XSPEC (version 12.10.1f; Arnaud 1996).

\footnotetext{
1 https://www.swift.ac.uk/user_objects/

2 http://hea-www.harvard.edu/AstroStat/BEHR/
}

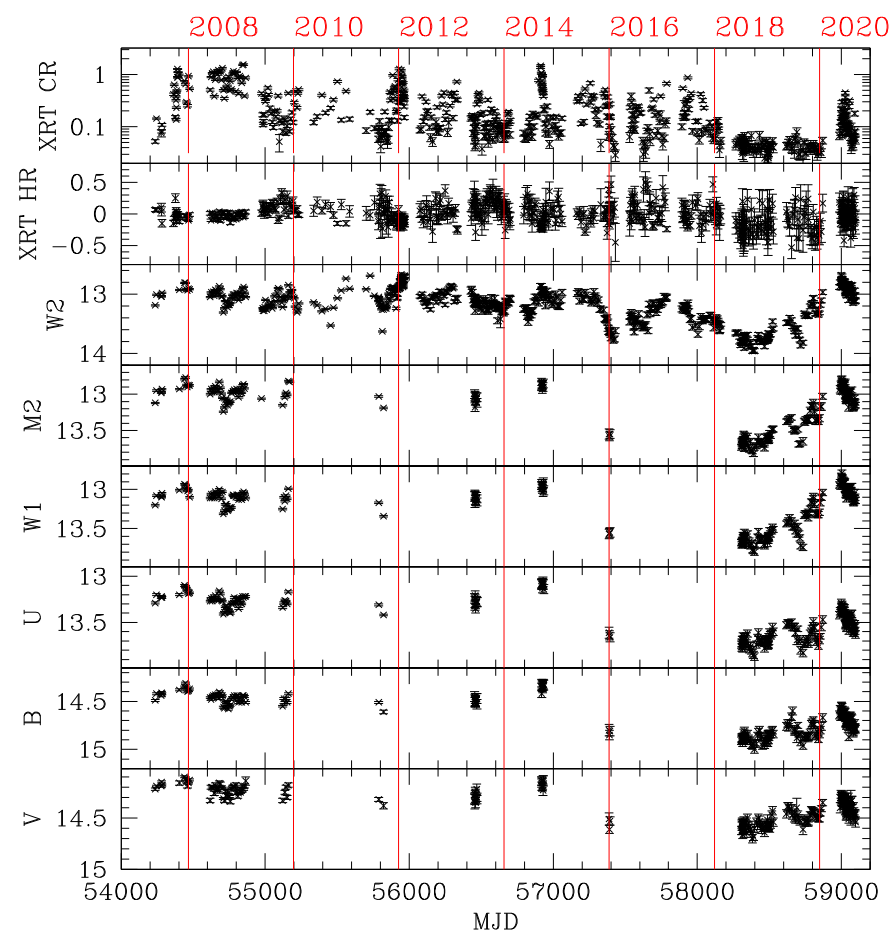

Fig. 1. Long-term Swift XRT and UVOT light curves of Mrk 335 from 2007 May to 2020 July. From top to bottom: XRT count rate, hardness ratio, and UVOT Vega magnitudes corrected for Galactic extinction. The vertical red lines mark the beginning of each second year from 2008 to 2020 .

\subsection{Swift UVOT}

Mrk 335 was observed with the UV-optical telescope (UVOT; Roming et al. 2005) either in W2 or in all six filters in order to obtain spectral energy distribution (SED) information of this rapidly varying AGN. Observations in each filter were coadded using the task uvotimsum. Source counts in all filters were then extracted in a circular region with a radius of $5^{\prime \prime}$, and the background was selected in a nearby region of radius $20^{\prime \prime}$. The background-corrected counts were then converted into VEGA magnitudes and fluxes based on the latest calibration as described in Poole et al. (2008) and Breeveld et al. (2010). The task uvotsource was used to measure the magnitudes and flux densities. A Galactic reddening correction was applied to the UVOT data, with a value of $E_{(B-V)}=0.035$ (Schlegel et al. 1998), with a correction factor in each filter according to Eq. (2) of Roming et al. (2009) and making use of the reddening curves of Cardelli et al. (1989).

\section{Results}

\subsection{Light curves and DCF analysis}

Figure 1 displays the long-term light curve of Mrk 335 in X-rays as well as in the optical/UV since the start of our Swift monitoring program in May 2007, when it was discovered in an unusually low X-ray flux state (Grupe et al. 2007). Figure 2 highlights the 2020 evolution in all bands.

Mrk 335 exhibited several short-duration X-ray flares over the last 13 years when it was in a low state overall. The new rise in X-ray flux after the long period of very low activity of more than two years is remarkable; it is unprecedented in Mrk 335. 


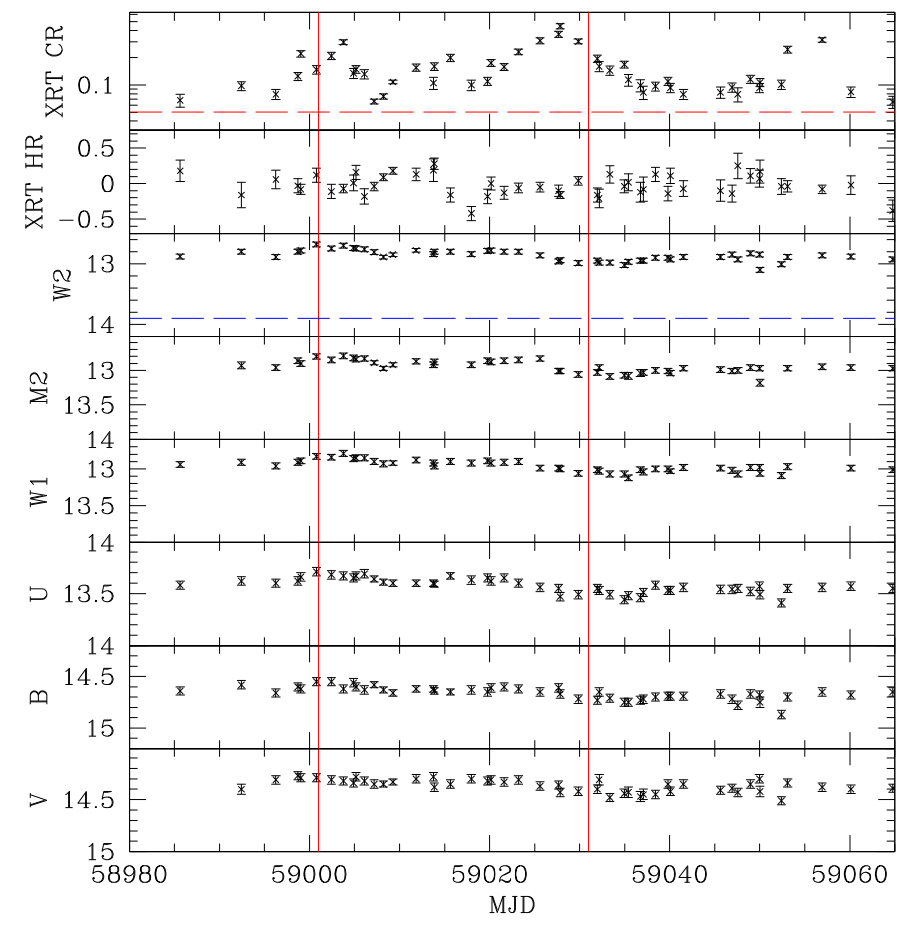

Fig. 2. Swift outburst light curve of Mrk 335 since 2020 May 16 (units as in Fig. 1). The horizontal dashed red line marks an X-ray count rate of $0.05 \mathrm{cts} \mathrm{s}^{-1}$ (the lowest recorded during the 2018-2019 low state was $0.03 \mathrm{cts} \mathrm{s}^{-1}$ ), while the horizontal dashed blue line marks the lowest value of the W2 magnitude measured with Swift (in 2018). The vertical red lines mark 2020 June 1 and July 1.

The systematic rise in the UV flux since about mid-2018 is even more remarkable.

Figure 2 shows the Swift results since we resumed our monitoring program on 2020 May 16. This light curve shows multiple rapid flare events in X-rays, including one rapid rise by a factor of $\sim 5$ within a week, and another rise by a factor of 2 within a day. Changes in the X-ray spectral shape as shown in the hardness ratio light curve are apparent. The XRT count rate before May 2020 was at a very low level around $0.05 \mathrm{cts} \mathrm{s}^{-1}$ for more than two years (and it is still variable, therefore not host dominated), and now (at the peak of the flare in late June 2020) it reached a value that is a factor of 10 higher. The brightest state in the UV (W2) was detected on 2020 May 31 with a Galacticreddening corrected magnitude of $12.68 \pm 0.04$. This is the highest state since the UV flare seen in December 2011.

In order to search for correlations and time delays between different bands during the 2020 outbursts, a discrete correlation function (DCF; Edelson \& Krolik 1988) analysis of the UVW2 and B and X-ray light curves was carried out following Gallo et al. (2018) with Monte Carlo confidence intervals. The $\mathrm{UV}$ band is closely correlated with the optical band, such that the optical is following and the UV leading by $\Delta t \simeq 1.5 \mathrm{~d}$ with a confidence interval of 3 to 0 days at the $99.99 \%$ level. However, no correlation between the UV and X-rays is found.

\subsection{X-ray spectral analysis}

Spectra were first fit with single power laws of photon index $\Gamma_{\mathrm{X}}$ adding Galactic absorption with a column density $N_{\mathrm{H}, \mathrm{Gal}}=$ $3.96 \times 10^{20} \mathrm{~cm}^{-2}$ (Kalberla et al. 2005). Because this model did not provide good spectral fits and left strong systematic residuals in all fits (Fig. 3), we then applied either an ionized or neutral
Table 1. X-ray spectral fit results of Mrk 335 (see Sect. 3.2).

\begin{tabular}{|c|c|c|c|c|c|}
\hline $\begin{array}{l}\text { Model } \\
(1)\end{array}$ & $\begin{array}{l}N_{\mathrm{H}} \\
(2) \\
\end{array}$ & $\begin{array}{l}\Gamma_{X} \\
(3) \\
\end{array}$ & $\begin{array}{c}k T \text { or } \log \xi \\
\text { (4) }\end{array}$ & $\begin{array}{c}\eta \\
(5) \\
\end{array}$ & $\begin{array}{c}\chi^{2} \text { red } \\
(6)\end{array}$ \\
\hline \multicolumn{6}{|c|}{ High state, 2020 June 23 - July 1} \\
\hline $\mathrm{pl}$ & & $2.49 \pm 0.06$ & & & 3.2 \\
\hline $\mathrm{zpcf} * \mathrm{pl}$ & $6.3_{-1.9}^{+3.3}$ & $2.95_{-0.14}^{+0.14}$ & & $0.86_{-0.05}^{+0.04}$ & 1.4 \\
\hline bbdy $+(\operatorname{zpcf} * \mathrm{pl}){ }^{(1)}$ & $4.3_{-1.1}^{+1.7}$ & $2.97 \pm 0.20$ & 0.15 & $0.88_{-0.05}^{+0.04}$ & 1.5 \\
\hline zxipcf* $*$ l & $16.1_{-4.9}^{+4.2}$ & $2.3_{-0.1}^{+0.1}$ & $2.1_{-0.1}^{+0.2}$ & $0.87_{-0.06}^{+0.07}$ & 1.1 \\
\hline \multicolumn{6}{|c|}{ Deep low state, 2019 May ${ }^{(2)}$} \\
\hline $\mathrm{pl}$ & & $2.34 \pm 0.20$ & & & 1.6 \\
\hline zpcf $*$ pl & $13.95_{-5.06}^{+8.62}$ & $3.07 \pm 0.21$ & & $0.94_{-0.06}^{+0.03}$ & 1.0 \\
\hline $\mathrm{pl}+$ bbdy & & $0.50_{-0.55}^{+0.51}$ & $0.15 \pm 0.02$ & & 0.9 \\
\hline
\end{tabular}

Notes. Columns are as follows: (1) Models: $\mathrm{pl}=$ powerlaw, bbdy = black body, zpcf $=$ partial covering absorber, and zxipcf $=$ ionized absorber model. (2) Absorption in units of $10^{22} \mathrm{~cm}^{-2}$; (3) power-law photon index; (4) $k T$ in units of $\mathrm{keV}$ of the black body, or ionization parameter $\log \xi$ of the ionized absorber; (5) covering factor; and (6) goodness of fit, reduced $\chi^{2}$. If no errors are reported, the quantity was fixed. For all fits the column density at $z=0$ was fixed to the Galactic value of $3.96 \times 10^{20} \mathrm{~cm}^{-2}$. (1) bbdy parameters fixed at low-state value. ${ }^{(2)}$ Fits using Cash statistics (Cash 1979).

partial covering absorber (Table 1). The single-component partial covering model consistently requires a lower column density and covering fraction at high state. Alternatively, the high-state spectrum is well fit by an ionized absorber. The 2019 lowstate spectrum can also be well described by a simple powerlaw plus black body model, where the black body is taken to be representative of a soft excess, which is dominated by a number of emission lines detected with the RGS in low state (Longinotti et al. 2019; Parker et al. 2019) and not individually resolved with Swift. We have added such a component (with its parameters fixed to the low-state values) when fitting the highstate spectrum, but it does not provide a significant change in the overall fit results. Based on the simple partial covering absorber fit to the 2020 high state, a (0.3-10) keV X-ray luminosity of $8.2 \times 10^{43} \mathrm{erg} \mathrm{s}^{-1}$ is obtained, or an X-ray Eddington ratio of $L_{\mathrm{X}} / L_{\text {Edd }} \approx 0.02$, while for the 2019 low state, the intrinsic X-ray luminosity is a factor of 1.6 lower when the same model description is adopted.

Spectral fits were also performed on a 2020 low state and intermediate state (Fig. 3). However, as Mrk 335 varies strongly during the 2020 time interval (unlike during the deep May 2019 low state), spectral fits are less reliable and still come with large errors, and at any given time interval a mix of cold and ionized absorbers may contribute. These cannot be disentangled with Swift, however. Applying the simple partial covering model to the 2020 low state, we find $N_{\mathrm{H}}=11.32_{-2.97}^{+4.62} \mathrm{~cm}^{-2}$, while for the intermediate state, we find $N_{\mathrm{H}}=4.32_{-0.88}^{+1.22} \mathrm{~cm}^{-2}$.

\subsection{Hardness ratio variability}

We inspected the long-term (since 2007) and short-term (2020) $H R$ variability of Mrk 335 (Fig. 4) including all data points until 2020 July 19. Three regimes are apparent: a softer-when-fainter pattern at lowest count rate $C R$, a softer-when-brigther trend at intermediate $C R$, and an almost constant $H R$ at highest $C R$. These three regimes have also been identified in other absorbed AGN that are intrinsically bright (e.g., Connolly et al. 2014). Results of a regression analysis (available in MIDAS) of the three variability regimes are shown in Fig. 4. A correlation analysis at $C R<0.1 \mathrm{cts} \mathrm{s}^{-1}$ gives $r_{\mathrm{s}}=+0.5, T_{\mathrm{S}}=8.7$, and $P<10^{-8}$ $(N=234)$. At $0.1<C R<0.25 \mathrm{cts} \mathrm{s}^{-1}, r_{\mathrm{s}}=-0.27, T_{\mathrm{S}}=-3.7$, 


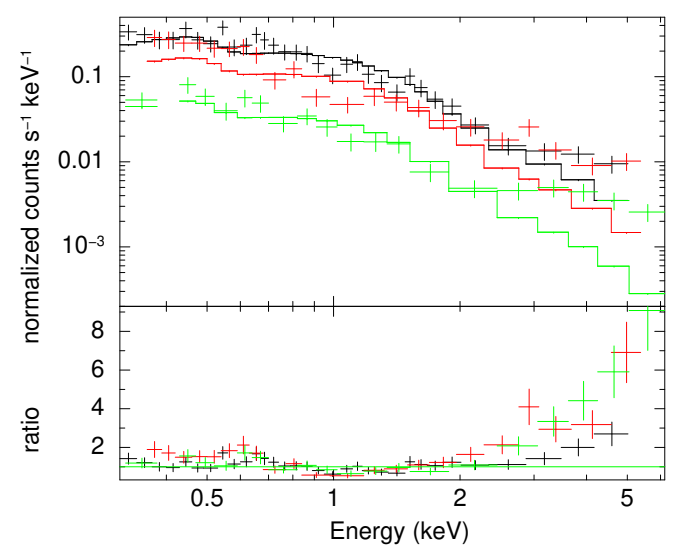

Fig. 3. Selected Swift spectra of Mrk 335 in 2020 May-July. The 2020 June high state is plotted in black, an intermediate state from June 2 and 3 in red, and a low state from 2020 (data from May 16, 23, and 27, and June 7) in green. All spectra are fit with a single power-law model with Galactic absorption, which displays strong fit residuals especially at low and high energies.

and $P=0.00014(N=177)$, while at $C R>0.25 \mathrm{cts} \mathrm{s}^{-1}$, $r_{\mathrm{S}}=-0.205, T_{\mathrm{S}}=-2.7$, and $P=0.0038(N=174)$, where $r_{\mathrm{S}}$ is the Spearman rank order correlation coefficient, $T_{\mathrm{S}}$ is the Student T-test, $P$ is the probability that the distribution is just random, and $N$ is the number of data points.

\subsection{Spectral energy distribution}

The SEDs during the high state in May-June 2020 in comparison with a low -state data set in May 2019 are shown in Fig. 5. The results show that the optical-UV spectrum has become bluer. While the W2-U color during the low state in 2019 was $-0.03 \pm 0.06$, it was $-0.61 \pm 0.06$ on 2020 May 31 . In X-rays, Mrk 335 has not only become brighter, but the spectrum has changed as well (see Sect. 3.2).

\section{Discussion}

The long-term Swift light curve of Mrk 335 reveals that its deepest minimum-state in the UV was reached in 2018, when the $\mathrm{X}$-ray emission was also at a deep and long-lasting low state, with little variability left in X-rays (Fig. 1). Except for one deviating epoch in 2019, and with some short-time variability superposed, the UV has been on a steady rise since its 2018 minimum, reaching and exceeding its former Swift peak value (2011) again in 2020 May-June (Fig. 1) $)^{3}$. Instead, the longterm X-ray light curve remained flat throughout 2018-2019, and we only detected a significant rise since 2020 May, with multiple peaks. Except that both reached a deep low-state in 2018 (Parker et al. 2019, which was relatively short-lived in the UV, however; Fig. 1), the variability observed with Swift in the $\mathrm{UV}$ and X-rays is not closely correlated on the timescale of days to months (see also Grupe et al. 2012; Gallo et al. 2018; Tripathi et al. 2020): multiple flares in the UV are not seen in $\mathrm{X}$-rays and vice versa, as confirmed by our DCF analysis of the 2020 data. This behavior is markedly different from that of other bright type 1 AGN (Shappee et al. 2014; Gardner \& Done 2017;

\footnotetext{
3 at no time during the Swift monitoring did the UV emission of Mrk 335 reach the highest brightness level recorded with IUE in the 1980s (e.g., Dunn et al. (2006), Fig. 1 of Grupe et al. (2008), Longinotti et al. (2013), Fig. 14 of Tripathi et al. (2020)).
}
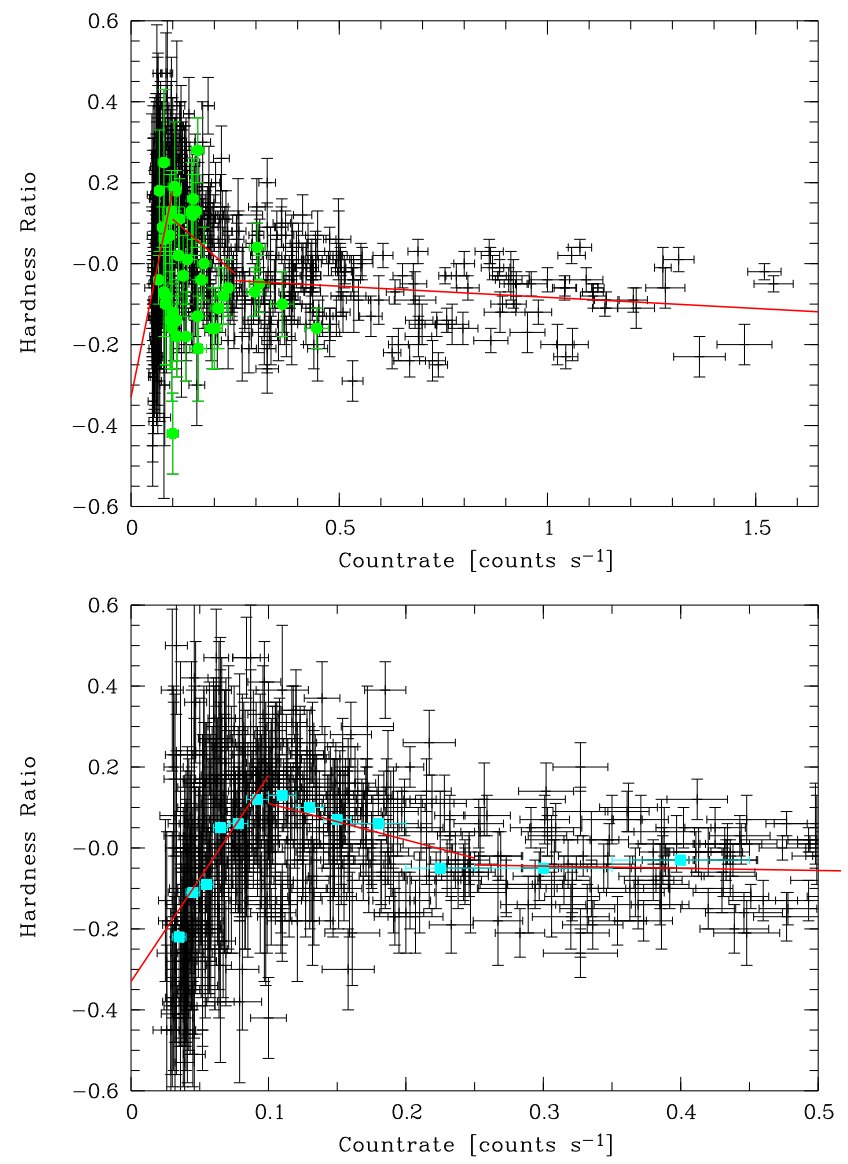

Fig. 4. Hardness ratio vs. X-ray count rate for Swift XRT data of Mrk 335 since 2007 (black). Three regimes are apparent: a softer-whenfainter trend at lowest count rate, a softer-when-brigther pattern at intermediate count rate, and a near-constant $\mathrm{HR}$ at highest count rate. Upper panel: full count-rate range. Green circles represent the 2020 outbursts. Lower panel: count-rate range up to $0.5 \mathrm{cts} \mathrm{s}^{-1}$. The red lines represent the fits to the three regimes (Sect. 3.3), and the blue squares mark the median in each bin.

Buisson et al. 2017; McHardy et al. 2018; Edelson et al. 2019) and demands an explanation (see below).

Several mechanisms are known to cause strong changes in the X-ray flux and spectral states of AGN: the first mechanism is changes in ionization state or geometry of ionized or neutral absorption that fully or partially covers the continuum source (e.g., Abrassart \& Czerny 2000; Turner et al. 2011; Leighly et al. 2015). Varying absorption is frequently seen in intermediate-type Seyfert galaxies whose variability timescales can be as short as hours or days, but can also occur in NLS1s (Risaliti et al. 2011) when the absorber is part of a clumpy accretion-disk wind or resides within the BLR. The second mechanism is changes in the X-ray reflection of photons off the (inner) accretion disk (Ross \& Fabian 2005). This model has frequently been applied to explain short-time spectral changes in type 1 Seyfert galaxies by changes in the location and geometry of the corona. Third, on longer timescales, changes in the accretion rate including disk instabilities can cause high or low states in AGN (e.g., Ross et al. 2018; Czerny 2019). This last mechanism has been favored to explain some of the extreme changinglook AGN that also switch their Seyfert types.

In Mrk 335, the variability of the UV band is not correlated with the X-ray band on the timescale of days to months (with the 


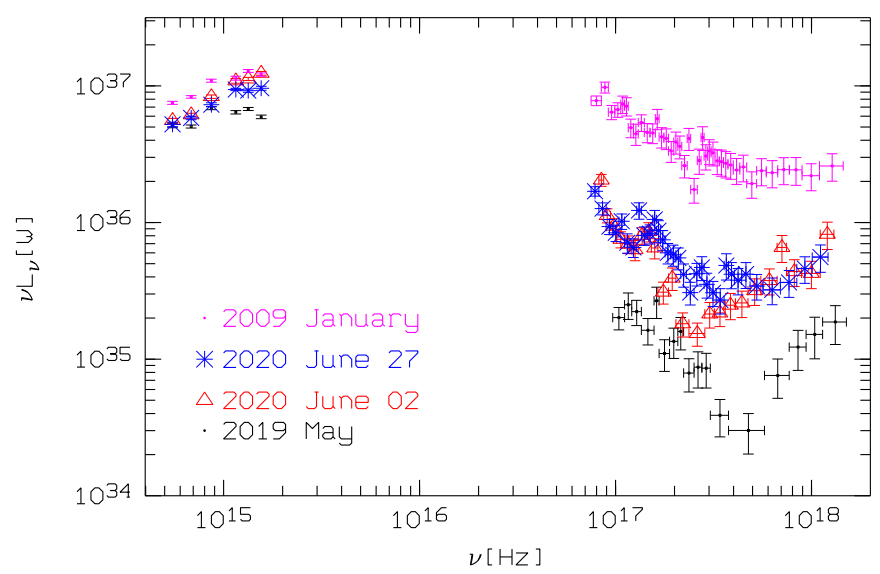

Fig. 5. Observed Swift SED of Mrk 335 during 2020 June 2, June 27, and the deep low-state of 2019 May. Note the X-ray flux deficit around (0.7-1.2) keV on June 2. For comparison, the Swift SED during the highest X-ray state from 2009 January is shown (purple).

exception of an epoch in 2014; Gallo et al. 2018). This rejects models that predict a close link between UV and X-rays: for instance, models where the observed X-rays are upscattered UV photons; or models where the UV is reprocessed emission from an X-ray corona heating the disk, which have successfully been applied to several well-observed AGN that show a close UV-Xray correlation (Shappee et al. 2014; McHardy et al. 2018); or models where UV and X-rays are due to synchrotron radiation (unlikely for Mrk 335 even though it is a radio emitter).

Furthermore, we can also exclude models of distant dusty absorbers that diminish the UV by extinction and simultaneously diminish the X-rays by absorption. However, dust-free absorption, arising in the accretion-disk region for instance in the form of a clumpy wind, is an efficient mechanism to cause X-ray spectral and flux changes while leaving the broad-band UV unaffected. And our spectral fits of a partial covering absorber consistently imply a decrease in covering factor and column density as the source brightens in X-rays. Our fit parameters of the ionization parameter, column density, and covering factor are consistent with predictions from models of clumpy disk winds (Takeuchi et al. 2013) that form via the RayleighTaylor and radiation-hydrodynamic instability (Shaviv 2001) and which predict variability timescales on the order of weeks.

A partial covering scenario can also explain why Mrk 335 only shows a mild trend of softer-when-brighter (Fig. 4) because a partial coverer would preserve the spectral shape as it leaks different fractions of the same intrinsic continuum when the covering factor changes. The softer-when-fainter pattern at the very lowest count rates is likely due to a soft X-ray component in the form of multiple emission lines (Longinotti et al. 2019; Parker et al. 2019) and a (distant) reflection component (Gallo et al. 2019) which become increasingly apparent as the primary continuum diminishes. While the X-ray covering factor obtained from the current and previous (intermediate to low state) observations of Mrk 335 is high ( $\sim 80-95 \%)$, a partial covering scenario for Mrk 335 is independently favored by previous UV observations that revealed variable absorption lines, including $\mathrm{Ly} \alpha$ at $20-30 \%$ covering fraction of the UV-emitting site (Longinotti et al. 2019; Parker et al. 2019).

There is other evidence that the X-rays we observe along our line of sight are not representative of the $4 \pi$ emission: optical reverberation mapping (Grier et al. 2012) has shown that the Balmer line $\mathrm{H} \beta$ and the high-ionization line HeII $\lambda 4686$ of
Mrk 335 follow the amplitude of the optical continuum variability $^{4}$ and not the higher amplitude seen in X-rays. While $R_{\max }=1.53$ in optical continuum variability, and $R_{\max }=1.55$ in HeII (Grier et al. 2012; where $R_{\max }$ is the ratio of maximum to minimum flux in each light curve), we measured during the same time interval $R_{\max }=5.44$ in X-rays.

Furthermore, no significant variability of the optical coronal lines in Mrk 335 was detected (Grupe et al. 2008) despite strong changes in observed X-rays. As those lines are driven by the EUV-to-soft-X-ray part of the SED, the observations indicate that the emission lines saw a different (less variable) continuum. An absorption scenario can also explain the lack of intrinsic variability of the photoionized soft X-ray emission lines (Parker et al. 2019), and is consistent with the lack of IR variability of Mrk 335 (Wright et al. 2010).

The question remains why Mrk 335 is no optical Seyfert-type changer ${ }^{5}$ despite its high amplitude of X-ray variability (a factor $\sim 50$ between highest and lowest state in our long-term Swift light curve). As described above, the emission-line regions seem to see a less variable EUV-X-ray SED. Furthermore, because the broad Balmer lines are intrinsically bright, even a drop of a factor of 2 still preserves the type 1 nature of Mrk 335 .

As Mrk 335 is a very bright AGN at high state, it may turn out to be a Rosetta stone in deciphering the various contributions of reflection, absorption, and intrinsic emission, which imprint their presence on AGN X-ray spectra. Following Mrk 335 closely as it emerges from low state will therefore provide us with an important opportunity of understanding the physical processes that drive long-term AGN state changes.

Acknowledgements. We would like to thank Brad Cenko for approving our Swift observing requests and the Swift team for carrying out our observations, and our anonymous referee for very useful comments. This work made use of data supplied by the UK Swift Science Data Centre at the University of Leicester. ALL acknowledges support from CONACyT grant CB-286316.

\section{References}

Abrassart, A., \& Czerny, B. 2000, A\&A, 356, 475

Arnaud, K. 1996, ASPC, 101, 17

Bianchi, S., Matt, G., Haardt, F., et al. 2001, A\&A, 376, 77

Breeveld, A. A., Curran, P. A., Hoversten, E. A., et al. 2010, MNRAS, 406, 1687 Buisson, D. J. K., Lohfink, A. M., Alston, W. N., \& Fabian, A. C. 2017, MNRAS, 464, 3194

Burrows, D. N., Hill, J. E., Nousek, J. A., et al. 2005, SSRv, 120, 165

Cardelli, J. A., Clayton, G. C., Mathis, J. S., et al. 1989, ApJ, 345, 245

Cash, W. 1979, ApJ, 228, 939

Choudhury, K., Nampalliwar, S., Abdikamalov, A. B., et al. 2019, ApJ, 879, 80 Connolly, S. D., McHardy, I. M., \& Dwelly, T. 2014, Proceedings of Swift: 10 Years of Discovery (SWIFT 10), Online at http://pos.sissa.it/ cgi-bin/reader/conf.cgi?confid=233

Czerny, B. 2019, Universe, 5, 131

Dunn, J. P., Jackson, B., Deo, R. P., et al. 2006, PASP, 118, 572

Edelson, R., \& Krolik, J. 1988, ApJ, 333, 646

Edelson, R., Gelbord, J., Cackett, E., et al. 2019, ApJ, 870, 123

Evans, P., Beardmore, A. P., Page, K. L., et al. 2007, A\&A, 469, 379

Gehrels, N., Chincarini, G., Giommi, P., et al. 2004, ApJ, 611, 1005

Gallo, L. 2018, Revisiting narrow-line Seyfert 1 galaxies and their place in the Universe (NLS1-2018), https://pos.sissa.it/cgi-bin/reader/ conf. cgi?confid=328, id.34

Gallo, L., Fabian, A., Grupe, D., et al. 2013, MNRAS, 428, 1191

Gallo, L., Wilkins, D. R., Bonson, K., et al. 2015, MNRAS, 446, 633

Gallo, L., Blue, D. M., Grupe, D., et al. 2018, MNRAS, 478, 2557

Gallo, L., Gonzalez, A. G., Waddell, S. G. H., et al. 2019, MNRAS, 484, 4287

4 The Balmer line emission is driven by the EUV beyond the Lyman limit, and the ionization potential of HeII is $54 \mathrm{eV}$.

5 As observed in an increasing number of other AGN (MacLeod et al. 2016). 
A\&A 643, L7 (2020)

Gardner, E., \& Done, C. 2017, MNRAS, 470, 3591

Grier, C. J., Peterson, B. M., Pogge, R. W., et al. 2012, ApJ, 744, L4

Grupe, D., Thomas, H.-C., \& Beuermann, K. 2001, A\&A, 367, 470

Grupe, D., Komossa, S., \& Gallo, L. 2007, ApJ, 668, L111

Grupe, D., Komossa, S., Gallo, L. C., et al. 2008, ApJ, 681, 982

Grupe, D., Komossa, S., Gallo, L., et al. 2012, ApJS, 199, 28

Grupe, D., Komossa, S., \& Gallo, L. 2020, ATel, 13757, 1

Hill, J. E., Burrows, D. N., Nousek, J. A., et al. 2004, SPIE, 5165, 217

Kalberla, P. M. W., McClure-Griffiths, N. M., Pisano, D. J., et al. 2005, A\&A, 440, 775

Kara, E., Fabian, A. C., Cackett, E. M., et al. 2013, MNRAS, 434, 1129

Komossa, S., Grupe, D., Schartel, N., et al. 2017, IAUS, 324, 168

Komossa, S. 2018, Revisiting narrow-line Seyfert 1 galaxies and their place in the Universe (NLS1-2018), https://pos.sissa.it/cgi-bin/reader/ conf. cgi? confid=328, id.15

Leighly, K. M., Cooper, E., Grupe, D., et al. 2015, ApJ, 809, L13

Longinotti, A. L., Krongold, Y., Kriss, G. A., et al. 2013, ApJ, 766, 104

Longinotti, A. L., Kriss, G., Krongold, Y., et al. 2019, ApJ, 875, 150

MacLeod, C. L., Ross, N. P., Lawrence, A., et al. 2016, MNRAS, 457, 389

Markarian, B. E. 1977, A\&A, 58, 139

McHardy, I. M., Connolly, S. D., Horne, K., et al. 2018, MNRAS, 480, 2881

Park, T., Kashyap, V. L., Siemiginowska, A., et al. 2006, ApJ, 652, 610
Parker, M. L., Wilkins, D. R., Fabian, A. C., et al. 2014, MNRAS, 443, 1723 Parker, M. L., Longinotti, A. L., Schartel, N., et al. 2019, MNRAS, 490, 683

Poole, T. S., Breeveld, A. A., Page, M. J., et al. 2008, MNRAS, 383, 627

Pounds, K., Stanger, V. J., Turner, T. J., et al. 1987, MNRAS, 224, 443

Risaliti, G., Nardini, E., Salvati, M., et al. 2011, MNRAS, 410, 1027

Roming, P. W. A., Kennedy, T. E., Mason, K. O., et al. 2005, SSRv, 120, 95

Roming, P. W. A., Koch, T. S., Oates, S. R., et al. 2009, ApJ, 690, 163

Ross, R. R., \& Fabian, A. C. 2005, MNRAS, 358, 24

Ross, N. P., Ford, K. E. S., Graham, M., et al. 2018, MNRAS, 480, 4468

Shappee, B. J., Prieto, J. L., Grupe, D., et al. 2014, ApJ, 788, 48

Shaviv, N. J. 2001, ApJ, 549, 1093

Schlegel, D. J., Finkbeiner, D. P., \& Davis, M. 1998, ApJ, 500, 525

Takeuchi, S., Ohsuga, K., \& Mineshige, S. 2013, PASJ, 65, 88

Tananbaum, H., Peters, G., Forman, W., et al. 1978, ApJ, 223, 74

Tripathi, S., McGrath, K. M., Gallo, L. C., et al. 2020, MNRAS, 499, 1266

Turner, T. J., Miller, L., Kraemer, S. B., et al. 2011, ApJ, 733, 48

Wilkins, D. R., \& Gallo, L. 2015, MNRAS, 449, 129

Wilkins, D. R., Gallo, L. C., Grupe, D., et al. 2015, MNRAS, 454, 4440

Wright, E. L. 2006, PASP, 118, 1711

Wright, E. L., Eisenhardt, P. R. M., Mainzer, A. K., et al. 2010, AJ, 140, 1868 
Appendix A: Log of observations

Table A.1. Summary of Swift observations of Mrk 335 in 2020 May - July.

\begin{tabular}{|c|c|c|c|c|c|c|c|c|c|c|c|}
\hline Target ID & Segment & Obs start (UT) & Obs end (UT) & $\operatorname{MJD}^{(1)}$ & $t_{\mathrm{XRT}}^{(2)}$ & $t_{\mathrm{V}}{ }^{(2)}$ & $t_{\mathrm{B}}{ }^{(2)}$ & $t_{\mathrm{U}}{ }^{(2)}$ & $t_{\mathrm{W} 1}{ }^{(2)}$ & $t_{\mathrm{M} 2}(2)$ & $t_{\mathrm{W} 2}{ }^{(2)}$ \\
\hline \multirow[t]{45}{*}{33420} & 240 & $2020-05-16$ 15:15 & $2020-05-16$ 15:25 & 58985.63905 & 637 & - & 66 & 86 & 79 & - & 266 \\
\hline & 241 & 2020-05-23 09:34 & 2020-05-23 09:42 & 58992.43683 & 480 & 38 & 38 & 38 & 78 & 105 & 154 \\
\hline & 242 & $2020-05-2706: 12$ & $2020-05-2706: 29$ & 58996.26405 & 995 & 79 & 79 & 79 & 157 & 249 & 315 \\
\hline & 243 & $2020-05-29$ 16:56 & $2020-05-29$ 17:10 & 58998.71010 & 834 & 70 & 70 & 70 & 141 & 171 & 280 \\
\hline & 244 & $2020-05-3000: 55$ & 2020-05-30 01:09 & 58999.04275 & 834 & 69 & 69 & 69 & 139 & 178 & 277 \\
\hline & 245 & $2020-05-31$ 18:19 & $2020-05-3118: 34$ & 59000.76809 & 897 & 73 & 73 & 73 & 145 & 205 & 292 \\
\hline & 246 & 2020-06-02 10:05 & 2020-06-02 10:15 & 59002.42337 & 587 & 45 & 45 & 45 & 90 & 149 & 181 \\
\hline & 247 & 2020-06-03 18:11 & 2020-06-03 18:28 & 59003.76332 & 999 & 81 & 81 & 81 & 163 & 226 & 328 \\
\hline & 248 & 2020-06-04 19:55 & 2020-06-04 23:11 & 59004.89769 & 974 & 77 & 77 & 77 & 155 & 215 & 311 \\
\hline & 249 & 2020-06-05 03:47 & 2020-06-05 04:01 & 59005.16214 & 844 & 69 & 69 & 69 & 137 & 192 & 273 \\
\hline & 250 & 2020-06-06 02:04 & $2020-06-0602: 20$ & 59006.09137 & 954 & 79 & 79 & 79 & 157 & 210 & 314 \\
\hline & 003 & $2020-06-1120: 23$ & 2020-06-11 20:40 & 59011.85493 & 1009 & 82 & 82 & 82 & 165 & 229 & 330 \\
\hline & 005 & 2020-06-13 18:36 & $2020-06-13$ 18:54 & 59013.78176 & 932 & 77 & 77 & 77 & 154 & 207 & 307 \\
\hline & 004 & 2020-06-13 20:23 & $2020-60-1320: 38$ & 59013.85416 & 904 & 74 & 74 & 74 & 146 & 208 & 293 \\
\hline & 006 & 2020-06-15 15:18 & 2020-06-15 $15: 31$ & 59015.64189 & 739 & 56 & 81 & 81 & 164 & - & 329 \\
\hline & 007 & 2020-06-17 23:05 & $2020-06-1723: 16$ & 59017.96548 & 629 & 50 & 50 & 50 & 101 & 143 & 203 \\
\hline & 008 & 2020-06-19 18:12 & 2020-06-19 18:29 & 59019.76407 & 989 & 83 & 83 & 83 & 163 & 216 & 328 \\
\hline & 009 & $2020-06-2003: 44$ & $2020-06-2003: 59$ & 59020.16048 & 889 & 73 & 73 & 73 & 146 & 196 & 293 \\
\hline & 010 & $2020-06-21 \quad 14: 54$ & $2020-06-21 \quad 15: 11$ & 59021.62666 & 974 & 81 & 81 & 81 & 160 & 213 & 322 \\
\hline & 011 & 2020-06-23 05:04 & 2020-06-23 05:21 & 59023.21670 & 1014 & 81 & 81 & 81 & 161 & 252 & 323 \\
\hline & 012 & 2020-06-25 14:18 & 2020-06-25 14:29 & 59025.59944 & 637 & 52 & 52 & 52 & 105 & 138 & 210 \\
\hline & 013 & 2020-06-27 15:39 & $2020-06-27$ 15:53 & 59027.65675 & 814 & 66 & 66 & 66 & 132 & 190 & 263 \\
\hline & 014 & $2020-06-27$ 20:22 & $2020-06-2720: 39$ & 59027.85434 & 991 & 81 & 81 & 81 & 161 & 229 & 323 \\
\hline & 015 & 2020-06-29 20:11 & $2020-06-29$ 20:27 & 59029.84626 & 946 & 77 & 77 & 77 & 153 & 219 & 307 \\
\hline & 016 & 2020-07-01 23:09 & 2020-07-01 23:24 & 59031.96965 & 864 & 71 & 71 & 71 & 142 & 194 & 283 \\
\hline & 017 & 2020-07-02 04:03 & 2020-07-02 04:11 & 59032.17113 & 492 & 39 & 39 & 39 & 79 & 107 & 157 \\
\hline & 018 & 2020-07-03 08:37 & 2020-07-03 08:52 & 59033.36409 & 864 & 70 & 70 & 70 & 140 & 202 & 279 \\
\hline & 019 & $2020-07-02 \quad 22: 51$ & 2020-07-04 23:06 & 59034.95704 & 887 & 72 & 72 & 72 & 143 & 206 & 287 \\
\hline & 020 & 2020-07-05 10:07 & 2020-07-05 10:15 & 59035.42399 & 477 & 37 & 37 & 37 & 76 & 110 & 151 \\
\hline & 021 & 2020-07-06 17:59 & 2020-07-06 18:07 & 59036.75174 & 482 & 37 & 37 & 37 & 75 & 116 & 148 \\
\hline & 022 & 2020-07-07 01:56 & 2020-07-07 02:05 & 59037.08344 & 524 & 40 & 40 & 40 & 80 & 135 & 159 \\
\hline & 023 & 2020-07-08 09:57 & 2020-07-08 10:14 & 59038.42019 & 1011 & 81 & 81 & 81 & 160 & 253 & 320 \\
\hline & 024 & 2020-07-09 19:32 & 2020-07-09 19:48 & 59039.81892 & 944 & 79 & 79 & 79 & 157 & 224 & 315 \\
\hline & 025 & 2020-07-01 01:51 & 2020-07-10 02:07 & 59040.08225 & 964 & 78 & 78 & 78 & 156 & 229 & 312 \\
\hline & 026 & $2020-07-11 \quad 12: 38$ & $2020-07-11 \quad 12: 54$ & 59041.53151 & 979 & 78 & 78 & 78 & 155 & 244 & 310 \\
\hline & 028 & 2020-07-05 15:34 & 2020-07-15 15:51 & 59045.65441 & 991 & 79 & 79 & 79 & 157 & 247 & 315 \\
\hline & 027 & 2020-07-16 21:56 & 2020-07-16 22:09 & 59046.91742 & 894 & 72 & 72 & 72 & 144 & 212 & 287 \\
\hline & 029 & 2020-07-17 13:56 & 2020-07-17 14:04 & 59047.58317 & 452 & 36 & 36 & 36 & 74 & 92 & 147 \\
\hline & 030 & 2020-07-18 23:17 & 2020-07-18 23:32 & 59048.97506 & 889 & 73 & 73 & 73 & 146 & 198 & 293 \\
\hline & 031 & 2020-07-19 23:13 & 2020-07-19 23:28 & 59049.97230 & 884 & 72 & 72 & 72 & 144 & 206 & 287 \\
\hline & 032 & 2020-07-20 00:46 & $2020-07-2000: 54$ & 59050.03457 & 450 & 35 & 35 & 35 & 70 & 104 & 141 \\
\hline & 033 & 2020-07-22 10:01 & 2020-07-22 10:16 & 59052.42186 & 864 & 73 & 73 & 73 & 145 & . & 290 \\
\hline & 034 & 2020-07-23 02:16 & $2020-07-23$ 02:26 & 59053.09802 & 649 & 51 & 51 & 51 & 102 & 153 & 205 \\
\hline & 035 & $2020-07-26 \quad 22: 37$ & $2020-07-2622: 54$ & 59056.94811 & 989 & 80 & 80 & 80 & - & 239 & 317 \\
\hline & 036 & 2020-07-30 03:10 & 2020-07-30 03:32 & 59060.13607 & 789 & 62 & 62 & 62 & 125 & 190 & 250 \\
\hline
\end{tabular}

Notes. ${ }^{(1)}$ The modified Julian date is given for the middle of the observation. ${ }^{(2)}$ All exposure times $t$ are given in seconds. 